\title{
Intravitreal bevacizumab (Avastin) in the treatment of macular edema secondary to retinal vein occlusion
}

\author{
Juan Carlos Mesa Gutiérrez \\ Luis Arias Barquet \\ Josep Maria Caminal \\ Mitjana \\ Sergi Prades Almolda \\ Nùria Planas Domènech \\ Octavi Pujol Goita \\ Marc Rubio Caso \\ Jorge Arruga Ginebreda \\ Department of Ophthalmology, \\ Hospital Universitari de Bellvitge, \\ L'Hospitalet de Llobregat, Barcelona, \\ Spain
}

Correspondence: Juan Carlos Mesa Gutiérrez

Department of Ophthalmology, Hospital Universitari de Bellvitge. Feixa Llarga s/n 08907. L'Hospitalet de Llobregat, Barcelona, Spain

Tel +34932607705

Fax +3493260798

Email juancarlosmesa@lycos.co.uk
Objective: To evaluate efficacy and safety of intravitreal injections of bevacizumab in the treatment of macular edema secondary to retinal vein occlusion (RVO).

Methods: Prospective study, noncomparative, interventional case series. Twelve consecutive patients (12 eyes) with macular edema associated with nonischemic retinal vein occlusion were treated with intravitreal bevacizumab $(1.25 \mathrm{mg})$. All subjects underwent standardized ophthalmic evaluation at baseline and at weeks 1, 4, 12, and 24, consisting of visual acuity (VA) measurement using ETDRS charts, and imaging with ocular coherence tomography evaluating changes in foveal thickness (FT) and macular volume (MV).

Results: The median age was 66 years $( \pm 4.16)$, and the median duration of symptoms was 4 months $( \pm 1.81)$. There were six cases of inferior branch vein occlusion and six cases of superior branch retinal vein occlusion. Mean VA improved from $1.32 \pm 0.24$ (logMAR values) at baseline to $0.8 \pm 0.15(\mathrm{p}=0.0003)$ at the 6 -month follow-up. The macular edema responded promptly, and a trend to restoration of normal macular anatomy was observed at by the seventh day. Mean FT improved from $615.50 \pm 116.29$ microns to $420 \pm 72.53$ microns $(\mathrm{p}=0.001)$, and the mean MV improved from $19.81 \pm 2.31 \mathrm{~mm} 3$ to $9.23 \pm 1.38(\mathrm{p}=0.0001)$ at the 6 -month follow-up.

Keywords: Bevacizumab, retinal vein occlusion, intravitreal injection, vascular endothelial growth factor

\section{Introduction}

Retinal vein occlusion (RVO) is the second most common retinal vascular disease, after diabetic retinopathy. Visual loss may result from ischemic damage and/or macular edema. Early treatment may be required to improve vision because longstanding macular edema results in irreversible photoreceptor damage (CRVO 1995). Intravitreal triamcinolone is a treatment option that has demonstrated promising short-term results for the management of macular edema associated with RVO (Mohammed et al 2007). A multicenter, randomized and controlled clinical trial (Standard Care Versus Corticosteroid for Retina Vein Occlusion Study) is currently underway.

Retinal vein occlusion is associated with varying amounts of retinal ischaemia and, consequently, increased concentrations of vascular endothelial growth factor (VEGF) (Hayreh 1983). Early case reports on bevacizumab showed an increase in visual acuity (VA) and a decrease in macular edema secondary to exudative age-related macular degeneration (AMD) and central retinal vein occlusion (CRVO) (Rosenfeld et al 2005a). A nonrandomized study of intravitreal bevacizumab in patients with CRVO resulted in reduced macular swelling and increased VA (Iturralde et al 2006). However, because a physiological level of vascular endothelial growth factor may be necessary to maintain the homeostasis of the retina, care might be required to avoid the possible negative consequences of a complete blockade of VEGF. 
These reports, along with results from preclinical and human studies that suggest a possible role of VEGF in RVO and the absence of a proven therapy, prompted us to investigate the effects of intravitreal bevacizumab injection in patients with macular edema associated with RVO.

\section{Methods}

\section{Study design}

This was a prospective, consecutive, noncomparative study that adhered to the Declaration of Helsinki and which was approved by our institutional review board. An intravitreal off-label bevacizumab injection was recommended. The Spanish Ministry of Health and Consumer Affairs approved compassionate use. Patients were fully informed verbally about the experimental nature of the treatment and they signed an informed consent form.

Cases were recruited from the Hospital Universitari de Bellvitge (Barcelona, Spain) from January-March 2007. Inclusion criteria were: 1) patients aged 50 or older 2) macular edema secondary to nonischemic RVO and 3) VA between 20/400 and 20/50 (Snellen equivalent). Exclusion criteria were: 1) history of retinal surgery or photocoagulation; 2) any history of a thromboembolic event; 3) bleeding disorders; and 4) use of anticoagulative medication other than aspirin. No patient refused treatment.

A comprehensive ophthalmic evaluation was performed; it included a medical history review, best corrected visual acuity testing (using ETDRS charts), slit-lamp biomicroscopy, dilated funduscopic examination using a 78-diopters lens and time domain ocular computed tomography (OCT) (Carl Zeiss Meditec, Dublin, CA, USA) that consisted of an acquisition protocol "Radial lines" (6 linear, $6 \mathrm{~mm}$ scans oriented at intervals of $30^{\circ}$ and centered on the foveal region). Macular maps were obtained using the "retinal thickness/volume" analysis protocol, and values for central foveal thickness (FT) and total macular volume (MV) were recorded. Follow-up examinations were scheduled at weeks $1,4,12$, and 24 post-injection, or on demand, if a decrease in VA was noted by the patient. These follow-up examinations used exactly the same procedures as those used in the baseline visit. The incidence of adverse events were monitored throughout the study. The effects of treatment, both on VA and on anatomical changes in the macula shown by OCT, were evaluated. There was no loss of follow-up.

\section{Treatment procedure}

Patients received an intravitreal dosage of bevacizumab of $1.25 \mathrm{mg}(0.05 \mathrm{~mL})$ at baseline and once every four weeks if
OCT indicated macular swelling (quantitatively characterized by a macular thickness larger than 250 microns in any of the six radial scans). All treatments were performed in the office using topical anaesthesia (tetracaine+oxibuprocaine) under sterile conditions. Bevacizumab was injected (using a 30-G needle) through the inferotemporal pars plana, $3.5 \mathrm{~mm}$ (pseudophakic) or $4 \mathrm{~mm}$ (phakic) posterior to the limbus. A drop of ofloxacine was applied to the affected eye immediately after the procedure and again every 6 hours for 4 days.

\section{Statistical data analysis}

All data were collected in an Microsoft Excel 2000 spreadsheet (Microsoft Corporation, Spain). For statistical analysis, the Wllcoxon test was performed. VA measurements were converted to $\log \mathrm{MAR}$ equivalents to perform analysis. $\mathrm{p}<0.05$ was considered significant.

\section{Results}

Twelve patients (seven women and five men) were included. The median age was 66 years and the median duration of symptoms prior to treatment was 4 months. Vein occlusion was located at the inferior branch in six patients and at the superior branch in the remaining six patients. There was a history of hypertension in four patients. All patients completed the 24-week follow-up examination; their baseline characteristics are summarized in Table 1.

Best corrected visual acuity (BCVA) data obtained over the course of the study are summarized in Table 2 . Evaluation of BCVA revealed significant improvement at all times compared with baseline. Mean VA improved from $1.32 \pm 0.24$ ( $\log$ MAR values) at baseline, to $0.8 \pm 0.15$ $(p=0.0003)$ at the 6-month follow-up. The macular edema responded promptly, and a reduction in the submacular fluid was observed at the seventh day. At baseline, the mean FT was $615.50 \pm 116.29$ microns; it declined to $420 \pm 72.53$ microns $(p=0.001)$. The mean MV improved from $19.81 \pm$ $2.31 \mathrm{~mm}^{3}$ to $9.23 \pm 1.38 \mathrm{~mm}^{3}(\mathrm{p}=0.0001)$ at the 6 -month follow-up. Mean changes in parameters recorded by OCT on weeks $1,4,12$, and 24 post-injection are summarized in Table 3. Overall, four patients were retreated: 2 patients received two consecutive injections of intravitreal bevacizumab, and two patients received three injections. No ocular or systemic adverse events were observed.

\section{Discussion}

This study demonstrates the early and clinically relevant benefits of bevacizumab injection for macular edema due to RVO. In this prospective case series, we found that intravitreal 
Table I Baseline characteristics

\begin{tabular}{|c|c|c|c|c|c|}
\hline Case, age, sex & Affected ye, localization & Duration (months) & IogMAR BCVA & OCT FT (microns) & OCT MV $\left(\mathrm{mm}^{3}\right)$ \\
\hline $\mathrm{I}, 64, \mathrm{~F}$ & OD, inferior & 2.5 & 0.63 & 664 & 12.91 \\
\hline $2,63, \mathrm{~F}$ & OD, inferior & 5 & 1.7 & 545 & 22.17 \\
\hline $3,75, M$ & OD, superior & 6 & 1.05 & 410 & 16.65 \\
\hline $4,57, M$ & OD, superior & 3 & 1.63 & 667 & 21.23 \\
\hline $5,67, \mathrm{~F}$ & OD, superior & 2.5 & 1.96 & 601 & 20.82 \\
\hline $6,66, \mathrm{~F}$ & OS, inferior & 2 & 0.62 & 763 & 11.9 \\
\hline $7,61 \mathrm{~F}$ & OD, superior & 3 & 1.6 & 495 & 21.16 \\
\hline $8,75, F$ & OD, superior & 4 & 1.04 & 630 & 15.64 \\
\hline $9,77, M$ & OS, superior & 4 & 1.62 & 239 & 20.22 \\
\hline $10,66, M$ & OS, inferior & 7 & 1.34 & 885 & 12.97 \\
\hline II, 59, M & OS, inferior & 6 & 1.3 & 498 & 11.2 \\
\hline $12,79, F$ & OS, inferior & 7 & 0.96 & 998 & 19.81 \\
\hline Median: 66 & & Median: 4 & Median: I.32 & Median: 615.50 & Median: 19.8I \\
\hline SD: 7,26 & & SD: I.8I & SD: 0.43 & SD: 205.547 & SD: 4.10 \\
\hline Confidence & & Cl: 1.02 & Cl: 0.24 & Cl: II6.29 & Cl:2.3I \\
\hline intervals $(\mathrm{Cl}): 4.16$ & & & & & \\
\hline
\end{tabular}

Abbreviations: F, female; M, male; SD, standard deviation; OD, right eye; OS, left eye; BCVA, best corrected visual activity; FT, foveal thickness; MV, macular volume.

injections of bevacizumab led both to a significant reduction of FT, as well as to an improvement of visual acuity in patients with RVO. A beneficial effect of intravitreal bevacizumab was observerd as early as the first week and over a 6-month follow-up period.

Our study supports the preliminary results of several recently published papers. The most detailed data on the natural history of CRVO were provided by the Central Vein Occlusion Study Group (CRVO 1995). Clinical outcomes of every new treatment option for CRVO must match with these data. In the natural course of CRVO, only $19 \%$ of patients with initial visual acuity of less than 20/200 had a chance of visual acuity of better than 20/200. Patients presenting with initial visual acuity between 20/200 and 20/50 had improvement to better than $20 / 50$ in $19 \%$ of cases; in $44 \%$ of cases acuity stayed between 20/200 and 20/50. The visual acuity of only $37 \%$ of patients became worse than $20 / 200$. Compared with these data, patients treated with intravitreal injections of bevacizumab showed much greater improvement. Priglinger and colleagues (2007) reported improvement in visual acuity from $20 / 250$ at baseline to $20 / 80$ at the 6-month follow-up $(\mathrm{p}<0.001)$ in a group of $46 \mathrm{CRVO}$ patients. Mean central retinal thickness decreased from $535 \pm 48$ microns at baseline to $323 \pm 116$ microns at the 6-month follow-up (Priglinger et al 2007). In a series of 30 CRVO patients followed for 6 months, Jason and colleagues (2007) reported improvement in VA from 20/394 at baseline to 20/313 at the 3-month follow-up, $(\mathrm{p}<0.05)$ and no significant changes after the fourth month. This indicates that bevacizumab represents an effective treatment option for CRVO and that the drug may improve the long-term prognosis of CRVO.

The intravitreal use of bevacizumab may provide anatomical and functional amelioration of the macula in patients with macular edema due to RVO. The electrical responses in the fovea and parafovea of the multifocal electroretinography recording depict a significant improvement at 1 and 3 months after the injection (Moschos and Moschos 2008).

Table 2 logMAR BCVA (baseline and I, 4 I2, and 24 weeks post-injection)

\begin{tabular}{|c|c|c|c|c|}
\hline $\begin{array}{l}\text { logMAR } \\
\text { BCVA } \\
\text { baseline }\end{array}$ & $\begin{array}{l}\text { logMAR } \\
\text { BCVA, } \\
\text { week I }\end{array}$ & $\begin{array}{l}\text { logMAR } \\
\text { BCVA } \\
\text { week } 4\end{array}$ & $\begin{array}{l}\text { logMAR } \\
\text { BCVA } \\
\text { week I } 2\end{array}$ & $\begin{array}{l}\text { logMAR } \\
\text { BCVA } \\
\text { week } 24\end{array}$ \\
\hline 0.63 & 0.35 & 0.17 & 0.23 & 0.43 \\
\hline 1.7 & 1.3 & 0.75 & 0.77 & 0.77 \\
\hline 1.05 & 1.01 & 1.03 & I & 0.89 \\
\hline 1.63 & 1.47 & 1.2 & 1.35 & 1.17 \\
\hline 1.96 & 0.4 & 0.43 & 0.61 & 0.53 \\
\hline 0.62 & 0.36 & 0.17 & 0.23 & 0.42 \\
\hline 1.6 & 1.2 & 0.75 & 0.78 & 0.76 \\
\hline 1.04 & 1.02 & 1.02 & I & 0.86 \\
\hline 1.62 & 1.48 & 1.3 & 1.36 & 1.19 \\
\hline 1.34 & I & 0.78 & 0.86 & 0.83 \\
\hline 1.3 & 1.28 & 1.24 & 1.28 & 1.25 \\
\hline 0.96 & 0.5 & 0.42 & 0.62 & 0.56 \\
\hline
\end{tabular}

Note: $p$ (baseline-week 24) $=0.0003$

Abbreviation: BCVA, best corrected visual acuity. 
Table 3 FT (microns) and MV (mm3) (baseline and I, 4, I2. and 24 week post-injection)

\begin{tabular}{|c|c|c|c|c|c|}
\hline Case I & Baseline & Week I & Week 4 & Week I 2 & Week 24 \\
\hline$\overline{\mathrm{FT}}$ & 664 & 220 & 166 & 167 & 189 \\
\hline MV & $12.9 \mid$ & 8.48 & 7.87 & 8.38 & 8.34 \\
\hline \multicolumn{6}{|l|}{ Case 2} \\
\hline FT & 545 & 440 & 165 & 481 & 654 \\
\hline MV & 22.17 & 6.96 & 6.64 & 9.33 & 8.35 \\
\hline \multicolumn{6}{|l|}{ Case 3} \\
\hline FT & 410 & 166 & 496 & 509 & 578 \\
\hline MV & 16.65 & 12.17 & 13.2 & 13.95 & 12.95 \\
\hline \multicolumn{6}{|l|}{ Case 4} \\
\hline FT & 667 & 565 & 459 & 500 & 475 \\
\hline MV & 21.23 & 17.32 & 15.16 & 15.69 & 14.63 \\
\hline \multicolumn{6}{|l|}{ Case 5} \\
\hline FT & 601 & 436 & 175 & 501 & 420 \\
\hline MV & 20.82 & 6.66 & 6.83 & 9.2 & 9.5 \\
\hline \multicolumn{6}{|l|}{ Case 6} \\
\hline FT & 763 & 400 & 170 & 456 & 432 \\
\hline MV & 11.9 & 7.47 & 6.86 & 7.37 & 10.56 \\
\hline \multicolumn{6}{|l|}{ Case 7} \\
\hline FT & 495 & 175 & 195 & 225 & 235 \\
\hline MV & 21.16 & 5.95 & 5.63 & 8.32 & 7.35 \\
\hline \multicolumn{6}{|l|}{ Case 8} \\
\hline FT & 630 & 220 & 460 & 335 & 342 \\
\hline MV & 15.64 & 11.16 & 12.1 & 12.94 & 10.55 \\
\hline \multicolumn{6}{|l|}{ Case 9} \\
\hline FT & 239 & 442 & 336 & 402 & 389 \\
\hline MV & 20.22 & 16.31 & 12.1 & 14.68 & 12.48 \\
\hline \multicolumn{6}{|c|}{ Case 10} \\
\hline FT & 885 & 296 & 450 & 425 & 420 \\
\hline MV & 12.97 & 6.25 & 10.91 & 10.55 & 8.95 \\
\hline \multicolumn{6}{|c|}{ Case II } \\
\hline FT & 498 & 345 & 450 & 356 & 352 \\
\hline MV & 11.2 & 10.72 & 5.82 & 10.97 & 7.55 \\
\hline \multicolumn{6}{|c|}{ Case 12} \\
\hline FT & 998 & 425 & 182 & 522 & 425 \\
\hline MV & 19.81 & 5.55 & 5.85 & 8.1 & 6.98 \\
\hline
\end{tabular}

Notes: $\mathrm{P}$ (baseline- week 24): $\mathrm{FT}=0.00 \mathrm{I} \mathrm{MV}=0.000 \mathrm{I}$

Abbreviations: FT, foveal thickness; MV, and macular volume.

Intravitreal injection of triamcinolone acetonide (TA) is another treatment option aimed to reduce macular edema after RVO. Several recent studies report favorable effects of intravitreal injection (4-20 mg) of TA on the course of RVO (Gregory et al 2006). The extent and duration of the effect of intravitreal injection of TA depends on the dose used and the presence of retinal ischemia (Jonas et al 2005). Repeated intravitreal injections of TA are possible; however, after repeated treatments, the effect on reduction of retinal thickness and increase in visual acuity are reduced (Boyd et al 2002; Kupperman et al 2007). Furthermore, although apparently improving the clinical outcome of RVO, repeated intravitreal injections of TA are associated with many potential complications, such as elevated intraocular pressure and cataract formation, which may ultimately decrease the long-term prognosis of RVO (Goff et al 2006). In contrast to intravitreal injection of TA, several injections of bevacizumab appear to have no drug-related complications. However, complications related to repeated intravitreal injections (eg, endophthalmitis, retinal tear, and lens trauma) must be taken into account (Jaissle et al 2006). Fortunately, none of these complications occurred in the present case series; this may be due to the thorough prophylactic, antiseptic regimen applied in our institution to minimize the likelihood of bacterial contamination.

The use of anti-VEGF agents in retinal disease has become increasingly common since the approval (in 2004 and 2006, respectively) of pegaptanib and ranibizumab for age-related maculopathy. These agents are currently being studied for their efficacy against macular edema due to RVO. The anti-VEGF agent most studied in regard to RVO is bevacizumab. Off-label intravitreal injection of bevacizumab was first reported in 2005 to represent a potential therapy for macular edema secondary to CRVO (Rosenfeld et al 2005b). Since then, several additional publications have reported favorable short-term results for reduction of macular edema and improvement of vision in patients with RVO (Spandau et 2006; Pau et al 2007).

Our results suggest a possible short-term benefit for macular architecture and VA; however, it is also clear that such benefits are transient. Continuous VEGF suppression may be required to sustain beneficial effects observed in the short term and the risks associated with multiple intravitreal injections need to be considered. Our data suggest that patients may require several injections to maintain efficacy. Three to four months after the most recent injection, worsening VA was detected in about half of the cases. The data in this study suggest that a single injection of intravitreal bevacizumab has a limited beneficial effect for approximately two months in most patients.

Although VEGF and its receptors represent potential targets for pharmacologic intervention, several important questions remain. Does VEGF play a role in the formation of vascular shunts across ischemic areas? Does continuous blockage of VEGF have a negative effect over the long term? Furthermore, recurrent macular edema may occur in patients 
with RVO following treatment with bevacizumab; in some cases, the recurrent macular edema may be more severe than the pre-treatment macular edema (a phenomenon known as "rebound" macular edema) (Matsumoto et al 2007).

The changes observed throughout the present study may provide important clues about drug effects and duration. Favorable macular changes, documented by OCT, were evident as soon as day 7 post-injection. While these improvements were maintained at post-injection week 4 , there was a clear tendency for macular edema to recur around week 12; this suggests, therefore, that reinjections might be considered at some point during this period when a $1.25 \mathrm{mg}$ dose regimen is used in setting of ischemic or nonischemic RVO.

The present study does have some limitations that must be recognized: there was no control group; we included only 12 patients and there was only a limited follow-up. However, the promising results reported here indicate that further studies of intravitreal bevacizumab injection for the management of ischemic or nonischemic RVO are justified. Future well-designed studies will help to establish the role of antiangiogenic therapy in the management of RVO.

\section{Disclosure}

The authors report no conflicts of interest in this work.

\section{References}

Boyd SR, Zachary I, Chakravarthy U, et al. 2002. Correlation of increased vascular endothelial growth factor with neovascularization and permeability in ischemic central vein occlusion. Arch Ophthalmol, 120:1644-50.

[CVRO] Central Vein Occlusion Study Group. 1995. Evaluation of grid pattern photocoagulation for macular edema in central vein occlusion. Ophthalmology, 102:1425-33.

Goff MJ, Jumper JM, Yang SS, et al. 2006. Intravitreal triamcinolone acetonide treatment of macular edema associated with central retinal vein occlusion. Retina, 26:896-901.

Gregori NZ, Rosenfeld PJ, Puliafito CA, et al. 2006. One-year safety and efficacy of intravitreal triamcinolone acetonide for the management of macular edema secondary to central retinal vein occlusion. Retina, 26:889-95.
Hayreh SS. 1983. Classification of central retinal vein occlusion. Ophthalmology, 458-74.

Iturralde D, Spaide RF, Meyerle CB, et al. 2006: Intravitreal bevacizumab (avastin) treatment of macular oedema in central retinal vein occlusion: a short-term study. Retina, 26:279-84.

Jaissle GB, Ziemssen F, Petermeier K, et al. 2006. Bevacizumab for treatment of macular edema secondary to retinal vein occlusion. Ophthalmoloe, 103:471-5.

Jason HSU, Kaiser R, Sivalingam A, et al. 2007. Intravitreal bevacizumab (Avastin) in central retinal vein occlusion. Retina, 27:1013-19.

Jonas JB, Akkoyun I, Kamppeter B, et al. 2005. Intravitreal triamcinolone acetonide for treatment of central retinal vein occlusion. Eur $J$ Ophthalmol, 15:751-8.

Kuppermann BD, Blumenkranz MS, Haller JA, et al. 2007. Randomized controlled study of an intravitreous dexamethasone drug delivery system in patients with persistent macular edema. Arch Ophthalmol, 125:309-17.

Matsumoto Y, Freund KB, Peiretti E. 2007. Rebound macular oedema following bevacizumab (Avastin) therapy for retinal venous occlusive disease. Retina, 27:426-31.

Mohamed Q, McIntosh RL, Saw SM, et al. 2007. Interventions for central retinal vein occlusion: an evidence-based systematic review. Ophthalmology, 114:507-19, 524.

Moschos MM, Moschos M. 2008. Intravitreao use of bevacizumab for macular edeam due to CRVO. A multifocal-ERG and OCT study. Doc Ophthalmol, 116:147-52.

Pai SA, Shetty R, Vijayan PB, et al. 2007. Clinical, anatomic, and electrophysiologic evaluation following intravitreal bevacizumab for macular edema in retinal vein occlusion. Am J Ophthalmol, 143:601-6.

Priglinger SG, Wolf AH, Kreutzer TC, et al. 2007. Intravitreal bevacizumab injections for treatment of central retinal vein occlusion. Six month results of a prospective trial. Retina, 27:1004-12.

Rosenfeld PJ, Fung AE, Puliafito CA. 2005a. Optical coherence tomography findings after an intravitreal injection of bevacizumab (Avastin) for macular oedema from central retinal vein occlusion. Ophthalmic Surg Lasers Imaging, 36:336-9.

Rosenfeld PJ, Moshfeghi AA, Puliafito CA. 2005b. Optical coherence tomography findings after an intravitreal injection of bevacizumab (avastin) for neovascular agerelated macular degeneration. Ophthalmic Surg Lasers Imaging, 36:331-7.

Spandau UH, Ihloff AK, Jonas JB. 2006. Intravirreal bevacizumab treatment of macular oedema due to central retinal vein occlusion. Acta Ophthalmol Scand, 84:555-6. 
